\title{
Aspectos destacados del Documento de Consenso Internacional de Expertos sobre Síndrome de Takotsubo
}

\author{
Highlights of the International Expert Consensus Document \\ on Takotsubo Syndrome
}

Dra. María Victoria Ramos

\section{Introducción}

El síndrome de takotsubo fue introducido en 1990 por Hikaru Sato y colaboradores, quienes publicaron cinco casos de pacientes con dolor torácico y electrocardiograma (ECG) característico de infarto agudo de miocardio (IAM), sin lesiones coronarias en la cineangiocoronariografía (CACG), y con un aspecto inusual del ventrículo izquierdo (VI) durante la sístole.

El término takotsubo deriva de la palabra japonesa utilizada para designar una vasija que permite capturar pulpos y remeda la forma del VI al final de la sístole. También se ha designado a esta patología como síndrome del corazón roto, cardiomiopatía de estrés y síndrome de balonamiento apical.

El síndrome de takotsubo se caracteriza por una anomalía transitoria de la motilidad parietal del VI que comparte aspectos comunes con el síndrome coronario agudo (SCA) (síntomas, anomalías del ECG, biomarcadores cardíacos elevados). Si bien se ha avanzado en el conocimiento de esta entidad, existen aún muchos aspectos sin aclarar. Aquí se resaltarán los últimos avances publicados este año por la Sociedad Europea de Cardiología.

\section{Epidemiología}

El reconocimiento de este síndrome se encuentra en aumento, correspondiendo a 5\%-6\% de las mujeres con sospecha de SCA con elevación del segmento ST. Su recurrencia se estima en $1,8 \%$ por paciente-año. Existe un franco predominio en el sexo femenino (90\%) y en mayores de 50 años (80\%), aunque se han reportado casos en niños.

\section{Síntomas y signos}

Los más comunes incluyen: dolor torácico agudo, disnea o síncope, que en un inicio son indistinguibles de un IAM. Su presencia varía de acuerdo con el desencadenante: en aquellos con gatillo emocional predomina el dolor torácico y las palpitaciones, mientras que cuando es secundario a estrés físico, las manifestaciones predominantes son las de la enfermedad subyacente (convulsiones, accidente cerebrovascular [ACV]).

\section{Criterios diagnósticos}

El diagnóstico de síndrome de takotsubo es desafiante por sus similitudes con el SCA. En el momento actual la CACG con ventriculografía continúa siendo el gold standar. Existen numerosos criterios diagnósticos desarrollados desde el año 2003, siendo los más ampliamente utilizados los de la Clínica Mayo. El grupo de consenso elaboró el InterTAK Diagnostic Criteria, basado en el conocimiento actual (tabla 1). Los principales cambios considerados son: 1) la inclusión del feocromocitoma como causa desencadenante (considerado criterio de exclusión previamente), 2) la presencia de enfermedad arterial coronaria (EAC) concomitante (reportada en 10\%-29\% de los casos), 3) la presencia de anormalidad en la motilidad parietal del VI que puede comprometer el territorio de una arteria coronaria (siendo más frecuente el de la descendente anterior), en cuyo caso la diferenciación con SCA o miocarditis requiere la realización de resonancia magnética (RNM) cardíaca.

Editora adjunta de la Revista Uruguaya de Cardiología.

Correspondencia: Dra. María Victoria Ramos. Correo electrónico: mariavictoriaramos@outlook.es

Recibido Ago 22,2018; aceptado Set 4, 2018 
Tabla 1. Criterios diagnósticos InterTAK (International Takotsubo Diagnostic Criteria)

Disfunción transitoria del VI (hipoquinesia, aquinesia, disquinesia) expresada como balonamiento apical, medio-ventricular, basal o focal. Puede existir compromiso del VD. La anomalía en la motilidad parietal habitualmente excede la distribución vascular epicárdica de una arteria coronaria.

Desencadenantes emocionales, físicos o combinados pueden preceder al síndrome, pero no es obligatorio.

Desórdenes neurológicos (HSA, ACV, convulsiones) y feocromocitoma pueden desencadenar el síndrome.

Nuevas alteraciones ECG (elevación o depresión del segmento ST, inversión de onda T, prolongación del intervalo QT). Pueden no existir cambios.

Elevación moderada de biomarcadores cardíacos (troponinas, creatin-kinasa) y significativo aumento del péptido natriurético cerebral.

La presencia de EAC significativa no excluye el diagnóstico de síndrome de takotsubo.

No existe evidencia de miocarditis infecciosa (se recomienda utilizar RNM cardíaca).

Afecta predominantemente a mujeres en la posmenopausia.

VI: ventrículo izquierdo; VD: ventrículo derecho; HSA: hemorragia subaracnoidea; ACV: accidente cerebrovascular; ECG: electrocardiograma; EAC: enfermedad arterial coronaria; RNM: resonancia magnética.

\section{Fisiopatología}

Si bien el mecanismo exacto no se conoce completamente, existe importante evidencia de que la estimulación simpática es esencial. El síndrome de takotsubo se ha asociado con aquellas circunstancias que generan un exceso de catecolaminas circulantes (estrés físico-emocional, feocromocitoma, enfermedades del sistema nervioso central). Se han demostrado niveles elevados de noradrenalina en el seno coronario de pacientes con esta afección. Existen múltiples hipótesis para explicar los patrones de balonamiento regional, a destacar: a) ruptura de placas ateroscleróticas excéntricas en pacientes con EAC no obstructiva; b) espasmo epicárdico multivaso; c) disfunción microcirculatoria coronaria secundaria a los efectos vasoconstrictores de las catecolaminas sobre los receptores $\alpha 1$ adrenérgicos; d) toxicidad directa de las catecolaminas sobre los cardiomiocitos (necrosis en banda de contracción por sobrecarga de calcio y disfunción contráctil secundaria a la disminución de la afinidad por dicho ión). Dado que la densidad de receptores $\alpha$ adrenérgicos es mayor en el ápex del VI (para compensar su menor inervación simpática), altos niveles de catecolaminas inducen cambios conformacionales en el receptor $\alpha 2$ resultando en inotropismo reducido y activación de la enzima óxido nítrico sintasa con generación de estrés oxidativo por radicales libres.

La reversibilidad de las anomalías de la motilidad parietal sugiere que existen mecanismos protectores capaces de preservar la integridad miocárdica. Fundamentalmente, la respuesta inotrópica negativa limita la injuria de forma similar al mecanismo de atontamiento y la activación de programas antiapoptóticos que permiten la supervivencia celular.

\section{Factores predisponentes}

Aunque la presencia de estresores mentales y físicos es universal, relativamente pocos pacientes desarrollan el síndrome de takotsubo, lo que implica que existen factores predisponentes. La frecuencia mayor en mujeres posmenopáusicas orienta a una influencia hormonal. Existe evidencia de que los estrógenos atenúan la vasoconstricción mediada por catecolaminas y disminuyen la respuesta simpática al estrés mental. Se han reportado casos familiares de síndrome de takotsubo que podrían sugerir la existencia de predisposición genética, probablemente por polimorfismos en los receptores adrenérgicos que comprometen su función. Los pacientes afectados presentan más comúnmente trastornos psiquiátricos como ansiedad y depresión; desórdenes neurológicos diversos (hemorragia subaracnoidea [HSA], convulsiones, ACV) pueden precipitar el síndrome, lo que sugiere una interacción cerebro-corazón.

\section{Gatillos}

La característica del síndrome de takotsubo es su asociación con un estresor previo. Si bien inicialmente se reportó al trauma emocional como el más frecuente, actualmente predominan los gatillos físicos, aunque las mujeres presentan más habitualmente un desencadenante emocional. Por otro lado, en un tercio de los pacientes no es posible determinar la existencia de un desencadenante. Los gatillos psicológicos incluyen múltiples emociones traumáticas, tanto positivas (fiestas sorpresa) como negativas (duelo, miedo, pánico, ira, ansiedad, problemas económicos, desastres naturales). Los gatillos físicos pueden relacionarse con actividad física, condiciones o procedimientos médicos. 


\section{Clasificación anatómica}

Pueden diferenciarse varios tipos de takotsubo basados en la localización de la anomalía de la motilidad: el típico balonamiento apical (el más común) y formas atípicas reconocidas recientemente (medio-ventricular, basal y regional) que presentan diferente fenotipo clínico: jóvenes, comorbilidades neurológicas, fracción de eyección del VI (FEVI) preservada, depresión del segmento ST en el ECG. La mortalidad intrahospitalaria es similar en las formas típicas y atípicas. El compromiso del ventrículo derecho que se observa en un tercio de los pacientes podría predecir peor evolución.

\section{Electrocardiograma}

El ECG inicial es anormal en la mayoría de los pacientes. En el registro InterTAK la frecuencia de alteraciones fue la siguiente: $44 \%$ elevación del segmento ST; 8\% depresión del segmento ST; 41\% inversión de onda $\mathrm{T}$, y bloqueo completo de rama izquierda en $5 \%$. Las alteraciones del ECG muestran una evolución temporal con resolución de la elevación del segmento ST, inversión progresiva de la onda T y prolongación del intervalo QT durante varios días, con normalización posterior en las siguientes semanas. La localización de la elevación del segmento ST e inversión de la onda $\mathrm{T}$ se corresponde con la región anatómica comprometida, habitualmente VI medio y apical, siendo estas alteraciones más notorias en DII y AVR y en precordiales, de V2 a V5. El compromiso de derivaciones inferiores (II, III, aVF) no es común. La presencia de ondas $\mathrm{Q}$ en derivaciones anteriores sin elevación del ST o inversión de onda T acompañante ocurre con cierta frecuencia y habitualmente es transitoria, consistente con atontamiento eléctrico. También puede observarse QRS de bajo voltaje, que traduce edema miocárdico.

\section{Score InterTAK}

El Registro Internacional de Takotsubo elaboró este score con la finalidad de evaluar la probabilidad de presentar el síndrome y adoptar estrategias de manejo en urgencias. Consta de siete parámetros que suman un total de 100 puntos (tabla 2). Existe alta probabilidad de síndrome de takotsubo cuando el paciente tiene un score $\geq 70$ puntos, mientras que si es $<70$ la probabilidad es baja o intermedia. Basados en este score se propone el siguiente algoritmo: pacientes con dolor torácico/disnea que presentan ECG sin elevación del segmento ST y score InterTAK $<70$ puntos deben realizarse CACG y ventriculografía, mientras que en los que presenten un score $\geq 70$ puntos podría

\begin{tabular}{|lr|}
\hline Tabla 2. Score InterTAK. & Puntos \\
\hline Parámetro & 25 \\
\hline Sexo femenino & 24 \\
Estrés emocional & 13 \\
Estrés físico & 12 \\
$\begin{array}{l}\text { Ausencia de depresión del segmento ST } \\
\text { (excepto aVR) }\end{array}$ & 11 \\
Alteraciones psiquiátricas & 9 \\
$\begin{array}{l}\text { Desórdenes neurológicos (HSA, ACV, } \\
\text { convulsiones) }\end{array}$ \\
Prolongación del intervalo QT & 6 \\
\hline $\begin{array}{l}\text { HSA: hemorragia subaracnoidea, ACV: accidente cerebrovas- } \\
\text { cular. }\end{array}$
\end{tabular}

considerarse inicialmente la realización de un ecocardiograma transtorácico (ETT).

En ausencia de las alteraciones típicas (balonamiento apical) se recomienda derivar al paciente para CACG. Los pacientes con dolor torácico/disnea y elevación del segmento ST en el ECG se consideran como SCA y deben enviarse para CACG urgente.

\section{Biomarcadores}

Todos los casos de takotsubo presentan necrosis miocárdica. Los valores pico de troponinas son habitualmente menores que en los SCA. La presencia de altos niveles al ingreso predice mal pronóstico. Se observa de forma frecuente un incremento sustancial del péptido natriurético cerebral y su pro hormona, vinculado al grado de activación simpática, concentraciones pico de proteína $\mathrm{C}$ reactiva y disfunción sistólica del VI. Se han estudiado otros biomarcadores, pero los resultados no son concluyentes.

\section{Técnicas de imagen}

El ETT es la técnica más utilizada para evaluar cambios en la función del VI y alteraciones en la motilidad regional, pudiendo identificarse diferentes variantes (apical, medio-ventricular, basal, focal). Permite, además, la identificación de complicaciones: obstrucción dinámica del tracto de salida del VI (TSVI) secundaria a la hiperquinesia basal compensadora, regurgitación mitral severa (funcional debido a desplazamiento de los músculos papilares), ruptura de la pared libre del VI, trombos en ápex y apéndice auricular izquierdo. Se identifican como predictores independientes de pronóstico adverso la FEVI baja, incremento en las presiones de llenado del VI y regurgitación mitral moderada a severa. La contractilidad del VI se recupera completamente 
en cuatro a ocho semanas. La angiografía cardíaca por tomografía puede ser una opción en pacientes que no puedan realizarse CACG por comorbilidades graves y en los individuos estables con baja probabilidad de SCA. En la fase subaguda puede utilizarse la RNM cardíaca, que además de permitir la identificación de las anomalías parietales cuantifica la función del VI y VD, evalúa la presencia de complicaciones y realiza la caracterización tisular del miocardio (edema, inflamación, necrosis, fibrosis). La presencia de realce tardío con gadolinio es predictora de pronóstico adverso. El edema miocárdico se visualiza frecuentemente en regiones con alteración de la función sistólica, secundario a inflamación, estrés parietal incrementado o isquemia transitoria.

\section{Complicaciones y pronóstico}

Aunque el síndrome de takotsubo se ha considerado una entidad benigna, recientes observaciones muestran incidencia de shock cardiogénico y muerte comparables a las de los SCA. Durante la fase aguda, cerca de $25 \%$ de los pacientes puede experimentar eventos adversos serios como inestabilidad hemodinámica y eléctrica. Se han identificado como predictores de resultado adverso: desencadenante físico, enfermedad neurológica o psiquiátrica, troponina inicial $>10$ veces el límite superior normal, y FEVI $<45 \%$ al ingreso. El sexo masculino tiene tres veces más riesgo de muerte y de eventos cardíacos y cerebrovasculares adversos. Las complicaciones más frecuentes durante el ingreso incluyen: insuficiencia cardíaca aguda (12\%-45\%), obstrucción del TSVI (10\%-25\%), regurgitación mitral (14\%-25\%) y shock cardiogénico (6\%-20\%). Menos frecuentemente se observa fibrilación auricular (5\%-15\%), trombo en VI (2\%-8\%), paro cardiorrespiratorio (4\%-6\%), bloqueo auriculoventricular completo (5\%), taquiarritmias y bradiarritmias (2\%-5\%), y defecto septal ventricular $(<1 \%)$.

Si bien existen pocos datos de sobrevida a largo plazo, se ha reportado recientemente que la mortalidad de los pacientes con takotsubo es similar a la de aquellos con EAC. A corto plazo, la presencia de arritmias ventriculares (taquicardia ventricular, torsades de pointes, fibrilación ventricular) constituye una causa frecuente de muerte que obliga al monitoreo en la fase subaguda (días 2 a 4), coincidiendo con inversión de las ondas $\mathrm{T}$ y prolongación del intervalo QT. Como consecuencia, se ha consi- derado el síndrome de takotsubo como una forma adquirida del síndrome de QT largo.

\section{Manejo terapéutico}

Dada la ausencia de estudios randomizados, el tratamiento de estos pacientes se basa en la experiencia clínica y en la opinión de expertos.

Partiendo de la dificultad en distinguir el síndrome de takotsubo de un SCA, los pacientes deben ser trasladados a un centro con servicio de hemodinamia y de cuidados cardiológicos intensivos. El tratamiento inicial es el mismo que el de los SCA e incluye ácido acetilsalicílico (AAS) y heparina, morfina y oxígeno si es necesario. En los individuos que se presentan con shock cardiogénico y balonamiento apical debe descartarse la presencia de obstrucción del TSVI, ya que las catecolaminas exógenas pueden empeorar la situación. El uso de betabloqueantes (BB), aunque supone una buena opción del punto de vista fisiopatológico, está contraindicado en caso de insuficiencia cardíaca severa, hipotensión y bradicardia, especialmente cuando el intervalo QT es mayor a $500 \mathrm{~ms}$. Los inhibidores de la enzima convertidora de angiotensina (IECA) y bloqueantes del receptor de angiotensina II (BRA) pueden facilitar la recuperación del VI. Se indican diuréticos en caso de edema pulmonar y nitroglicerina para reducir las presiones de llenado interventricular (contraindicados en caso de obstrucción del TSVI). El tratamiento de las complicaciones no difiere del empleado en los pacientes con SCA.

A largo plazo el uso de IECA y BRA se ha asociado con mejoría en la sobrevida, sin observarse beneficio con la terapia BB ni prevención de recurrencias (cercanas a 5\%). Cuando existe concomitancia con EAC, se recomienda el uso de AAS y estatinas.

\section{Bibliografía}

1. Ghadri JR, Wittstein IS, Prasad A, Sharkey S, Dote K, Akashi YJ, et al. International expert consensus document on takotsubo syndrome (Part I): clinical characteristics, diagnostic criteria, and pathophysiology. Eur Heart J 2018;39(22):2032-46. doi: 10.1093/eurheartj/ehy076

2. Ghadri JR, Wittstein IS, Prasad A, Sharkey S, Dote K, Akashi YJ, et al. International expert consensus document on takotsubo syndrome (Part II): diagnostic workup, outcome, and management. Eur Heart J 2018;39(22):2047-62. doi: 10.1093/eurheartj/ehy077 